\title{
Mimetics of brain-derived neurotrophic factor loops $I$ and 4 are active in a model of ischemic stroke in rats
}

\author{
Tatyana A Gudasheva' \\ Polina Povarnina' \\ Ilya O Logvinov \\ Tatyana A Antipova ${ }^{2}$ \\ Sergey B Seredenin ${ }^{3}$ \\ 'Department of Medicinal Chemistry, \\ ${ }^{2}$ Department of Neuroprotective \\ Pharmacology, ${ }^{3}$ Department of \\ Pharmacogenetics, VV Zakusov \\ Institute of Pharmacology, \\ Moscow, Russia
}

\author{
This article was published in the following Dove Press journal: \\ Drug Design, Development and Therapy \\ 2 November 2016 \\ Number of times this article has been viewed
}

\begin{abstract}
Background: Two dimeric dipeptides, bis-(N-monosuccinyl-L-seryl-L-lysine)hexamethylenediamide (GSB-106) and bis-(N-monosuccinyl-L-methionyl-L-serine) heptamethylenediamide (GSB-214), were designed based on the brain-derived neurotrophic factor (BDNF) loop 4 and loop $1 \beta$-turn sequences, respectively. Earlier, both of these dipeptides were shown to exhibit neuroprotective activity in vitro $\left(10^{-5}-10^{-8} \mathrm{M}\right)$. The present study aimed to investigate the mechanisms of action of these peptides and their neuroprotective activity in an experimental stroke model.
\end{abstract}

Methods: We used western blot and HT-22 hippocampal neuronal cell line to investigate whether these peptides induced phosphorylation of the TrkB receptor and the AKT and ERK kinases. Rat middle cerebral artery occlusion (MCAO) was used as a stroke model. GSB-106 and GSB-214 were administered intraperitoneally $\left(0.1 \mathrm{mg}\left(1.3 \times 10^{-7} \mathrm{~mol}\right) / \mathrm{kg}\right) 4$ hours after MCAO and daily for 7 days. The cerebral infarct volumes were measured with 2,3,5-triphenyltetrazolium chloride staining 21 days after MCAO.

Results: Both compounds were shown to elevate the TrkB phosphorylation level while having different post-receptor signaling patterns. GSB-106 activated the PI3K/AKT and MAPK/ERK pathways simultaneously, whereas GSB-214 activated the PI3K/AKT only. In experimental stroke, the reduction of cerebral infarct volume by GSB-106 $(\sim 66 \%)$ was significantly greater than that of GSB-214 ( $28 \%$ reduction), which could be explained by the fundamental role of the MAPK/ERK pathway in neurogenesis and neuroplasticity. Notably, between these two dipeptides, only GSB-106 exhibited antidepressant activity, as was found previously.

Conclusion: The results provided support for the beneficial pharmacological properties of BDNF loop 4 mimetic GSB-106, thereby suggesting a potential role for this dipeptide as a therapeutic agent.

Keywords: brain-derived neurotrophic factor, mimetic, PI3K/AKT, MAPK/ERK, ischemic stroke

\section{Background}

Stroke is one of the leading causes of death and long-term disability worldwide, and no drugs are available for promoting recovery after a stroke has occurred.

There is a growing body of evidence indicating the role of brain-derived neurotrophic factor (BDNF) in recovery after stroke. BDNF has been reported to reduce infarct volumes and improve neurological outcomes in rodents subjected to cerebral ischemia. ${ }^{1-3}$ Increased BDNF levels after stroke during the acute (hours to days) and subacute periods (days to weeks) are beneficial for improving lost function. ${ }^{4}$ The mechanisms of the neuroprotective capability of BDNF include anti-apoptotic effects, ${ }^{5}$
Correspondence: Tatyana A Gudasheva Department of Medicinal Chemistry, VV Zakusov Institute of Pharmacology, Baltiyskaya str 8, 125315 Moscow, Russia Tel +74956012246

Fax +7 49915। |26।

Email tata-sosnovka@mail.ru 
modulation of local inflammatory processes, ${ }^{6}$ promotion of neurogenesis, ${ }^{7}$ improvement of synaptogenesis, and synaptic plasticity mechanisms. ${ }^{8,9}$

BDNF exerts its main biological actions through TrkB receptors. Binding of BDNF to TrkB leads to the activation of various intracellular signaling pathways, including the $\mathrm{PI} 3 \mathrm{~K} / \mathrm{AKT}$ and MAPK/ERK pathways, which are the most critical for the biological effects of BDNF. ${ }^{5}$ The PI3K/AKT pathway has been associated mainly with neuroprotection. ${ }^{10}$ The MAPK pathway is also involved in neuroprotection and plays a pivotal role in the mechanisms of neurogenesis and neuroplasticity. ${ }^{11,12}$

Unfortunately, BDNF itself does not have favorable druglike characteristics, ie, a limited half-life and poor blood-brain barrier penetration, thus motivating the search for alternative approaches for enhancing BDNF signaling in the brain.

The dimeric dipeptide mimetics of BDNF loops 1 and 4 were designed and synthesized at the Zakusov Institute of Pharmacology (Moscow, Russia) ${ }^{13}$ (RU Patent No 2410392, 2010; US Patent Application No US 2015/0111828 A1). The dimeric dipeptide bis-(N-monosuccinyl-L-seryl-L-lysine) hexamethylenediamide (GSB-106) was designed based on the BDNF loop $4 \beta$-turn sequence -Asp93-Ser94-Lys95-Lys96-, which is the most exposed fragment and therefore might play a major role in the interaction of BDNF with the receptor. We included the central fragment of the $\beta$-turn, Ser94-Lys95, in the dipeptide composition. The residue Asp93 was substituted by its bioisostere, a succinic acid residue, and Lys96 was substituted by an amide group. The purpose of these two substitutions was to stabilize the $\beta$-turn conformation and to increase the resistance of the compound to peptidases. Because BDNF interacts with TrkB in the homodimer form, we linked two $\beta$-turn mimetics using a hexamethylene diamine spacer. The dimeric dipeptide bis-(N-monosuccinyl-L-methionyl-L-serine) heptamethylenediamide (GSB-214) was designed analogously to GSB-106, based on the BDNF loop $1 \beta$-turn.

It has been shown in vitro, using the HT-22 hippocampal neuronal cell line, that GSB-106 and GSB-214 exerted BDNF-like neuroprotective activity $\left(10^{-5}-10^{-8} \mathrm{M}\right) \cdot{ }^{13}$ Maximal neuroprotective effects were observed at concentrations of $10^{-8} \mathrm{M}$ (GSB-106) and $10^{-7} \mathrm{M}$ (GSB-214); therefore, these concentrations were used for further in vitro experiments. Because there is well-documented evidence of the involvement of BDNF in the pathogenesis of depression, we studied the antidepressant properties of GSB-106 and GSB-214. It was shown that the mimetic of the BDNF loop 4 GSB-106 exhibited antidepressant activity in the forced swimming test in mice, while the mimetic of the BDNF loop $1 \mathrm{GSB}-214$ did not. ${ }^{13}$ The most effective dose of GSB-106 in vivo was $0.1 \mathrm{mg}\left(1.3 \times 10^{-7} \mathrm{~mol}\right) / \mathrm{kg}$, administered intraperitoneally (ip).

In the present study, it was established that GSB-106 and GSB-214 activated the TrkB receptor and that they each had different post-receptor signaling patterns. GSB-106 increased the levels of ERK and AKT kinase phosphorylation, whereas GSB-214 only increased the level of AKT phosphorylation. Further, we tested the hypothesis that GSB-106 and GSB-214 would improve functional recovery after experimental stroke induced by occlusion of the middle cerebral artery in rats. Treatments with GSB-106 or GSB-214 caused significant reductions in brain infarct size and improvement of neurological outcomes, with GSB-106 demonstrating greater in vivo efficacy than GSB-214. The greater effects of GSB-106 could be explained by the fundamental role of the MAPK/ ERK pathway in neurogenesis and neuroplasticity, ${ }^{11,12}$ which are key factors in post-stroke recovery. Hence, GSB-214, which activated only the PI3K/AKT pathway, showed less pharmacological activity in this model.

\section{Methods \\ Drugs and reagents}

The dimeric dipeptides GSB-106 ((bis-(N-monosuccinylL-seryl-L-lysine)hexamethylenediamide ( $\mathrm{Tm}=143^{\circ} \mathrm{C}-$ $145^{\circ} \mathrm{C},[\alpha]_{\mathrm{D}}{ }^{20}=-24.7^{\circ}(c=0.4 \%$; dimethylformamide $)$ ) and GSB-214((bis-(N-monosuccinyl-L-methionyl-L-serine) heptamethylenediamide $\left(\mathrm{Tm}=160^{\circ} \mathrm{C}-162^{\circ} \mathrm{C},[\alpha]_{\mathrm{D}}{ }^{20}=-21.75^{\circ}\right.$ $(c=0.4 \% ; \mathrm{MeOH}))$ were synthesized at the Zakusov Institute of Pharmacology (Moscow, Russia), as described previously. ${ }^{13}$

2,3,5-triphenyltetrazolium chloride (TTC) and Nembutal were obtained from Sigma-Aldrich (St Louis, MO, USA). Dulbecco's Modified Eagle's Medium was purchased from HyClone Laboratories (Logan, UT, USA). Fetal bovine serum was obtained from Gibco (Langley, OK, USA). Glutamine was purchased from ICN Pharmaceuticals, Inc. (Costa Mesa, CA, USA). Poly-D-lysine was purchased from BD Biosciences (San Jose, CA, USA). DC protein assay was purchased from Bio-Rad (Hercules, CA, USA). Anti-TrkA, anti-pTrkA, anti-AKT1/2/3, antiphospho-AKT $1^{s 473} / 2^{s 472} / 3^{s 474}$ anti-ERK1/2, anti-phospho ERK1/2 $2^{\mathrm{Y} 204}$ antibodies, and enhanced chemiluminescence kits were obtained from Santa Cruz Biotechnology (Dallas, TX, USA). Anti- $\beta$-actin antibodies and horseradish peroxidase-conjugated antibodies were purchased from Abcam (Cambridge, MA, USA). Formalin was purchased from Ecros (Saint Petersburg, Russia). GSB-106 and 
GSB-214 were dissolved in water. Then solvents were diluted in culture media in equivalent amounts.

\section{Cell cultures}

The mouse hippocampal cell line HT-22 was maintained in Dulbecco's Modified Eagle's medium, 10\% fetal bovine serum, and $2 \mathrm{mM}$ glutamine in $5 \% \mathrm{CO}_{2}$ and $95 \%$ air at $37^{\circ} \mathrm{C}$ and was passaged by trypsinization. Cells were placed on six-well plastic plates previously treated with poly-D-lysine $\left(5 \mu \mathrm{g} / 1 \mathrm{~cm}^{2}\right)$ at a plating density of $200 \times 103$ cells per well. The cultures were maintained in a humidified atmosphere of $5 \% \mathrm{CO}_{2}$ and $95 \%$ air at $37^{\circ} \mathrm{C}$.

\section{Western blot analysis}

HT-22 cells were collected 15, 30, and 60 min after incubation with GSB-106 $\left(10^{-8} \mathrm{M}\right)$, GSB-214 $\left(10^{-7} \mathrm{M}\right)$, or BDNF $\left(10^{-9} \mathrm{M}\right),{ }^{14}$ and protein was extracted for western blot analysis. Samples were homogenized in a lysis buffer (50 mM Tris$\mathrm{HCl}$, pH 7.5, 5 mM EDTA, 1 mM DTT, 1\% Triton X-100 supplemented with protease and phosphatase inhibitor cocktail) and were incubated on ice for $5 \mathrm{~min}$ and then centrifuged $\left(13,000 \mathrm{rpm}, 10 \mathrm{~min}\right.$, at $\left.4^{\circ} \mathrm{C}\right)$. Protein levels of the supernatant lysates were measured using Bio-Rad DC protein assay. Proteins were separated on $10 \%$ sodium dodecyl sulfate polyacrylamide gel electrophoresis gel and were blotted for $1 \mathrm{~h}(15 \mathrm{~V})$ onto a polyvinylidene fluoride (PVDF) membrane. The membranes were incubated at $4^{\circ} \mathrm{C}$ overnight with the following primary antibodies: anti-pTrkB, anti-phospho$\mathrm{AKT}^{\text {S473 }}{ }^{\text {, anti-phospho-AKT2 }}{ }^{\text {S472, }}$, anti-phospho-AKT3 ${ }^{\text {S474 }}$, anti-phospho ERK1/2 ${ }^{\mathrm{Y} 204}$, anti-TrkB, anti-ERK1/2, and antiAKT1/2/3. All of the antibodies were used at 1:1,000 final dilutions in $0.5 \%$ non-fat dry milk in tris-buffered saline $/ 0.5 \%$ Tween (TBST). Equal load was confirmed using anti-actin ( $1: 5,000$ in $0.5 \%$ non-fat dry milk in TBST). The membranes were washed with TBST and were incubated with horseradish peroxidase-conjugated secondary antibody (1:20,000 in 0.5\% non-fat dry milk in TBST). Secondary antibodies tagged to peroxidase were used to visualize immunoreactive bands using enhanced chemiluminescence kits.

\section{Transient middle cerebral artery occlusion (MCAO) model and experimental protocol}

All of the experiments were performed in accordance with the requirements of Directive 2010/63/EU of the European Parliament and were approved by the Institutional Animal Care and Use Committee of Zakusov Institute of Pharmacology (Moscow).
Thirty-nine (250-320 g) male outbred rats (Stolbovaya Breeding Center, Moscow, Russia) were used. Focal cerebral ischemia was induced by transient MCAO using a modification of the intraluminal filament model originally described by Longa et al. ${ }^{15}$ Briefly, the rats were anesthetized with an ip injection of Nembutal $(60 \mathrm{mg} / \mathrm{kg})$. The right common carotid artery, internal carotid artery, and external carotid artery were surgically exposed. A Nylon suture $(0.25 \mathrm{~mm}$ in diameter $)$ with a silicon-coated tip was inserted from the external carotid artery into the internal carotid artery and then to the circle of Willis to occlude the origin of the middle cerebral artery. After $1 \mathrm{~h}$ of MCAO, the suture was carefully removed to induce reperfusion. Sham-operated rats $(n=8)$ underwent identical surgery except that the suture was not inserted. The body temperature was maintained at $37.0^{\circ} \mathrm{C} \pm 0.5^{\circ} \mathrm{C}$ using a heating pad during the surgery. The rats that died due to anesthesia were excluded $(n=7)$. The rats receiving MCAO that did not have any neurological deficits when awakening were excluded $(\mathrm{n}=5)$. The remaining animals were randomly divided into 3 groups: a MCAO group with water treatment (MCAO, $\mathrm{n}=7$ ) and two MCAO groups treated with GSB-106 (MCAO + GSB-106, n=6) or GSB-214 (MCAO + GSB-214, n=6).

GSB-106 and GSB-214 (dissolved in distilled water) were administered ip at a dose of $0.1 \mathrm{mg}\left(1.3 \times 10^{-7} \mathrm{~mol}\right) / \mathrm{kg} 4 \mathrm{~h}$ after MCAO and daily for 7 days. This dose was chosen as the most pharmacologically active dose, based on preliminary in vivo studies. ${ }^{13}$ The sham group and MCAO group were treated with distilled water.

\section{Neurological score assessment Limb-placing test}

Neurological functions were evaluated 3 and 7 days after MCAO (day of surgery was day 0) using the limb-placing test, ${ }^{16}$ a modified version of the test described by De Ryck et al (1989). ${ }^{17}$ This test assessed the forelimb and hind limb responses to tactile and proprioceptive stimulation and consisted of seven limb-placing tasks. The following scores were used to detect impairment of the forelimb and hind limb: 2 points, the rat performed normally; 1 point, the rat performed with a delay of more than $2 \mathrm{~s}$ and/or incompletely; and 0 point, the rat did not perform the task. Both sides of the body were tested. The maximum possible score for the sham-operated rats for each side of the body was 14 .

\section{Cylinder test}

At 7 days after MCAO, the cylinder test was used to assess the asymmetry of forelimb usage. ${ }^{18}$ The rats were placed individually inside a transparent cylinder $(20 \mathrm{~cm}$ in diameter 
and $30 \mathrm{~cm}$ in height) and were recorded for $5 \mathrm{~min}$ and analyzed using a video recorder with slow motion capability. The numbers of contacts by both forelimbs and by either the impaired (contralateral to the lesion) or non-impaired (ipsilateral to the lesion) forelimb were counted, and the index for preference was obtained: (contralateral contacts + $1 / 2$ bilateral contacts $) /($ total contacts $) \times 100 \%{ }^{18}$

\section{Evaluation of cerebral infarct volume}

The cerebral infract volumes measured with TTC staining were used to describe the severity of cerebral ischemia. The animals were deeply anesthetized with $3.5 \%$ chloral hydrate and then decapitated following the completion of a neurologic assessment 21 days after MCAO. The brains were removed rapidly, frozen in $-20^{\circ} \mathrm{C}$ for $10 \mathrm{~min}$ and then sectioned coronally into $2 \mathrm{~mm}$ thick slices. The brain slices were incubated with $2 \% \mathrm{TTC}$ at $37^{\circ} \mathrm{C}$ for $30 \mathrm{~min}$. Stained slices were fixed in $10 \%$ formalin solution. The slices were digitalized on a flatbed scanner at 2,400 dpi. The infarct volumes were measured using the ImageJ (National Institutes of Health, Bethesda, MD, USA) image analysis software program. The total infarct volume for each brain was calculated by summation of unstained areas of the subsequent slices and multiplying by the thickness $(2 \mathrm{~mm})$.

\section{Statistical analysis}

Values are expressed as the mean \pm standard error of the mean or as the median (interquartile interval). Non-parametric statistics were used to test for statistical significance. The Mann-Whitney $U$-test was used for single comparisons. The Kruskal-Wallis test, followed by the pair-wise MannWhitney $U$-test with Bonferroni's correction, was used to compare three or more groups. $P$-values $<0.05$ were considered to indicate statistically significant effects.

\section{Results}

\section{Both dipeptides, GSB-I06 and GSB-2 I4, activate TrkB receptors}

The dipeptides GSB-106 $\left(10^{-8} \mathrm{M}\right)$ and GSB-214 $\left(10^{-7} \mathrm{M}\right)$ added to HT-22 hippocampal neurons induced the elevation of TrkB receptor phosphorylation, similar to BDNF $\left(10^{-9} \mathrm{M}\right)$ (Figure 1).

\section{GSB-106 and GSB-21 4 exhibit different patterns of PI3K/AKT and MAPK/ERK activation}

ERK or AKT activation by GSB-106 $\left(10^{-8} \mathrm{M}\right)$ and GSB-214 $\left(10^{-7} \mathrm{M}\right)$ was assessed using the ERK1/2 and AKT1/2/3 phosphorylation assays. Western blot densitometry analysis revealed that $\mathrm{AKT} 1 / 2 / 3$ phosphorylation was increased after stimulation of the cells by GSB-106 (10-8 M), GSB-214 $\left(10^{-7} \mathrm{M}\right)$, or BDNF $\left(10^{-9} \mathrm{M}\right)$ (Figure 2$)$.

GSB-106 and BDNF induced the phosphorylation of ERK1/2. There was no activation of ERK1/2 proteins by GSB-214 at any time point (Figure 3). These data suggested that GSB-214 selectively activates PI3K/AKT signaling, whereas GSB-106 activates both the PI3K/AKT and MAPK/ ERK pathways.

\section{GSB-106 and GSB-2I4 improve sensorimotor performance on the limb- placing test after MCAO}

Animals subjected to ischemia showed an increase in contralateral (left) limb placing deficits on the limb-placing test (Figure 4). The median limb-placing scores for left fore/hind limbs in this group were 9 (interquartile range, 7-10) and 6.5 (interquartile range, 5-7) at days 3 and 7 after MCAO, respectively. No deficits in ipsilateral limb placing in animals with cerebral ischemia were observed (data not shown). The sham-operated animals had no impairments in limb behavior at any time period, and their score was 14 for each side of the body (data not shown). Treatment with GSB-106 or GSB-214 significantly improved neurologic outcomes at days 3 and 7 after MCAO $(P<0.05)$.

\section{GSB-106 reduces forelimb use asymmetry on the cylinder test after MCAO}

To determine the extent of lateralizing motor deficits caused by stroke, the cylinder-rearing test was performed 7 days after MCAO. In this test, the sham-operated rats did not show any paw preference (Figure 5). After stroke, the rats had reduced left forelimb (contralateral) use, compared to the sham group. There was an overall significant effect of stroke on ipsilateral forelimb use. Treatment with GSB-106 completely reversed the deficits in contralateral limb use produced by MCAO (Figure 5). The effects of GSB-214 treatment were not statistically significant.

\section{GSB-106 and GSB-2I 4 decrease infarct volume after MCAO}

GSB-106 and GSB-214 administration significantly decreased infarct volumes 21 days after MCAO. The median infarct volume, as determined by TTC staining (Figure 6), was $90 \mathrm{~mm}^{3}$ (interquartile range, $73-121 \mathrm{~mm}^{3}$ ) in the GSB-106 treated rats and $194 \mathrm{~mm}^{3}$ (interquartile range, $124-222 \mathrm{~mm}^{3}$ ) 

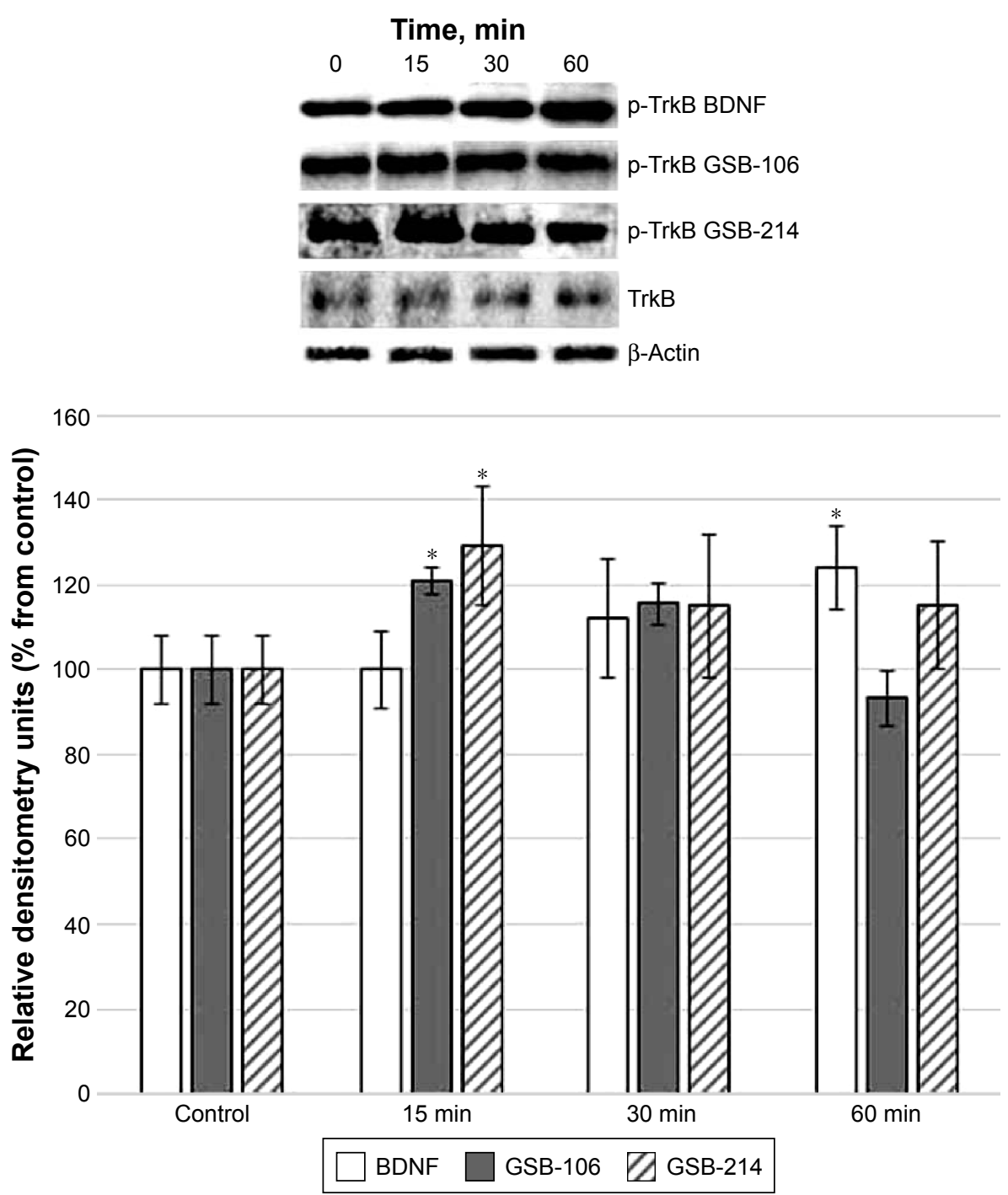

Figure I Western blot analysis of TrkB phosphorylation in HT-22 hippocampal neurons at different times (I5, 30, and 60 min) after incubation with GSB-I06 (I0-8 M), GSB-2I $4\left(10^{-7} \mathrm{M}\right)$, or BDNF $\left(10^{-9} \mathrm{M}\right)$.

Notes: Bar graphs represent the intensities of phosphorylated TrkB quantified by scanning densitometry of blots, and these intensities are expressed as percentages relative to controls. Total non-phosphorylated TrkB arbitrary densitometric units were analyzed separately against $\beta$-actin. Data are presented as the mean \pm SD of 5 independent experiments. Differences from the controls were significant at $* P<0.05$ (Mann-Whitney $U$-test).

Abbreviations: BDNF, brain-derived neurotrophic factor; GSB-I06, bis-(N-monosuccinyl-L-seryl-L-lysine) hexamethylenediamide; GSB-214, bis-(N-monosuccinyl-Lmethionyl-L-serine) heptamethylenediamide; SD, standard deviation; min, minutes.

in the GSB-214 treated rats, which was significantly $(P<0.05)$ less than in the saline treated control rats, which had a median infarct volume of $268 \mathrm{~mm}^{3}$ (interquartile range, $243-280 \mathrm{~mm}^{3}$ ) (Figure 6). Thus, the infarct volume was markedly decreased by $\sim 66 \%$ following GSB-106 administration and by $\sim 28 \%$ following GSB-214 administration.

\section{Discussion}

Thus, the dimeric dipeptide mimetics of the BDNF loops 1 and 4, GSB-214 and GSB-106, respectively, were shown to reduce cerebral infarct volume and to improve neurological function after transient $\mathrm{MCAO}$ in rats.
We found previously that GSB-214 and GSB-106 exerted neuroprotective activity in vitro. In the current study, we established that both compounds elevated the level of TrkB receptor phosphorylation, but they had different patterns of post-receptor signal transduction. The mimetic of BDNF loop 4 GSB-106 increased the levels of ERK and AKT kinase phosphorylation, whereas the mimetic of BDNF loop 1 GSB-214 increased the level of AKT phosphorylation only. Notably, dimeric dipeptide mimetics of nerve growth factor (NGF) loops 1 and 4 demonstrated the opposite patterns of post-receptor signal transduction, as we reported earlier. ${ }^{19}$ The mimetic of NGF loop 4, bis-(N-succinyl-L-glutamyl-L-lysine) 


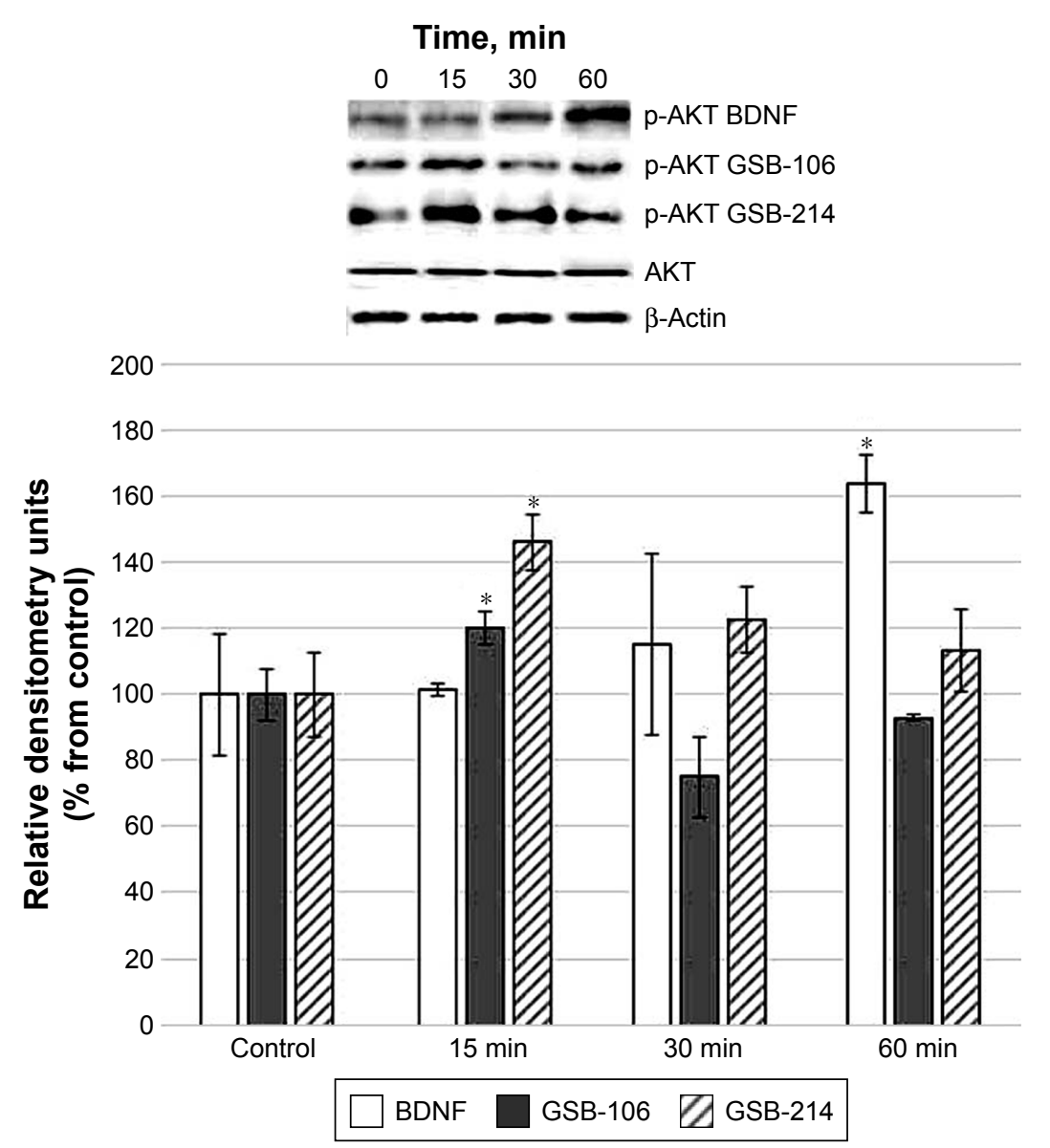

Figure 2 Western blot analysis of AKT phosphorylation in HT-22 hippocampal neurons at different times (I5, 30, and 60 min) after incubation with GSB-106 (I0-8 M), GSB-2I4 (10-7 M), or BDNF $\left(10^{-9} \mathrm{M}\right)$.

Notes: Anti-phospho-AKTI ${ }^{5473}$, anti-phospho-AKT2 ${ }^{5472}$, and anti-phospho-AKT3 ${ }^{5474}$ were used. Bar graphs represent the intensities of phosphorylated AKT quantified by scanning densitometry of blots, and they are expressed as percentages relative to controls. Total non-phosphorylated GSB-I06, hexamethylenediamide and; GSB-2I4, heptamethylenediamide arbitrary densitometric units were analyzed separately compared to $\beta$-actin. Data are presented as the mean \pm SD of 5 independent experiments. Differences from the controls were significant at $* P<0.05$ (Mann-Whitney $U$-test).

Abbreviations: BDNF, brain-derived neurotrophic factor; GSB-106, bis-(N-monosuccinyl-L-seryl-L-lysine) hexamethylenediamide; GSB-2I4, bis-(N-monosuccinyl-Lmethionyl-L-serine) heptamethylenediamide; SD, standard deviation.

hexamethylenediamide (GK-2), selectively activated the $\mathrm{PI} 3 \mathrm{~K} / \mathrm{AKT}$ signaling pathway, while the mimetic of NGF loop 1, bis-(N-aminocarbonyl-glycyl-L-lysine)hexamethylenediamide (GK-6), activated both the PI3K/AKT and MAPK/ERK pathways. ${ }^{19}$

In experimental stroke, GSB-106 demonstrated greater neuroprotective efficacy than GSB-214. The reduction of cerebral infarct volume by GSB-106 ( 66\%) was significantly greater than the effects on lesions by GSB-214 ( $28 \%$ reduction). The neuroprotective activity of GSB-106 was similar to that of BDNF, which decreased cerebral infarct volume by as much as $65 \%-70 \%$ in experimental models of forebrain ischemia. ${ }^{4,20}$ Both BDNF mimetics improved sensorimotor functions on the limb-place test with almost equal efficiency, but only GSB-106 statistically significantly reduced forelimb use asymmetry on the cylinder test. These differences in efficacy might reflect differences in the patterns of signal transduction. GSB-214 activated the PI3K/AKT pathway only, which is mainly associated with neuroprotection. ${ }^{10}$ GSB-106 activated the MAP-kinase pathway along with the PI3K/AKT pathway, with the former playing a pivotal role in the mechanisms of neurogenesis and neuroplasticity. ${ }^{11,12}$ Previously, we reported that the dimeric dipeptide mimetic of NGF loop 4, which selectively activates the PI3K/AKT pathway, reduced brain infarct volume by approximately $18 \%$ in a model of MCAO in rats. ${ }^{21}$ Our results showed that selective activation of the PI3K/AKT pathway could reduce brain infarct volume and could improve functional recovery after stroke. However, activation of both the PI3K/AKT and MAPK/ERK pathways led to more therapeutic efficacy, possibly through the stimulation of neurogenesis and neuroplasticity, which are the two types of cellular events implicated in recovery after stroke. Indeed, GSB-106 was found to stimulate hippocampal neurogenesis in adult mice. ${ }^{22}$ 
Time, $\min$

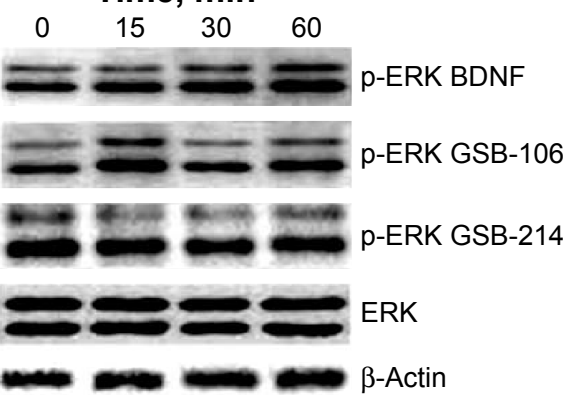

250

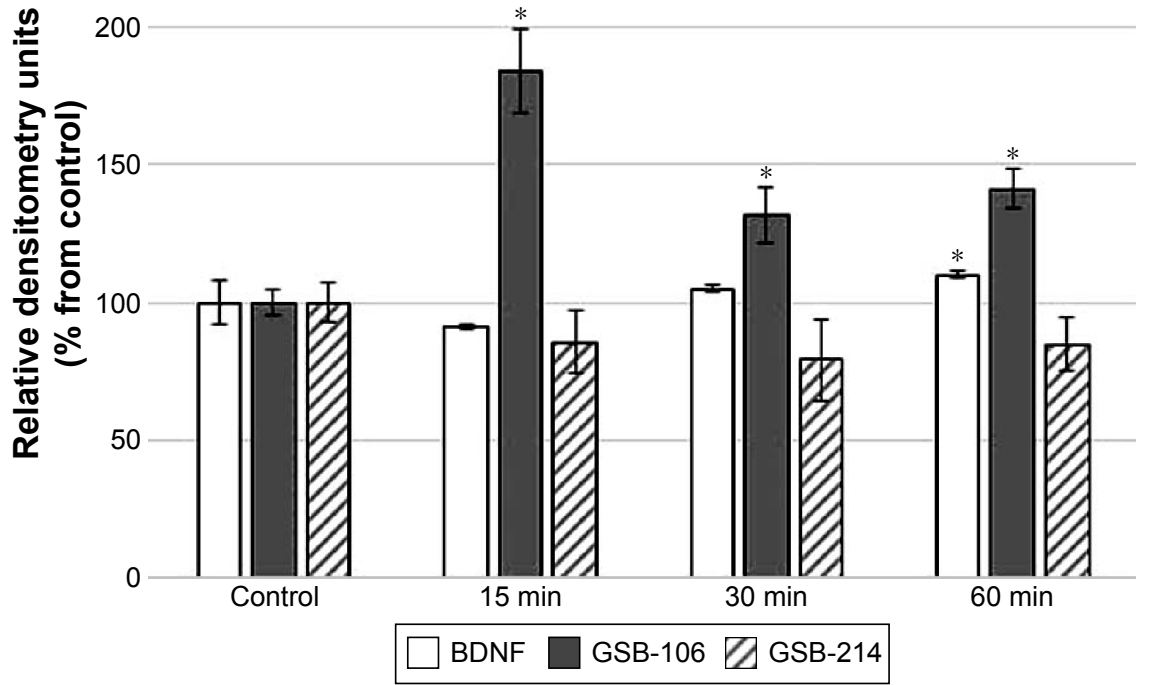

Figure 3 Western blot analysis of ERK phosphorylation in HT-22 hippocampal neurons at different times (15, 30, and 60 min) after incubation with GSB-106 (10-8 M),

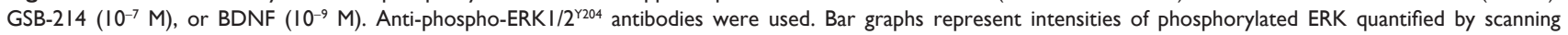
densitometry of blots, and these intensities are expressed as percentages relative to controls.

Notes: Total non-phosphorylated ERK arbitrary densitometric units were analyzed separately compared to $\beta$-actin. The data are presented as the means \pm SD of five independent experiments. Differences from the controls were significant at $* P<0.05$ (Mann-Whitney U-test).

Abbreviations: BDNF, brain-derived neurotrophic factor; ERK, extracellular signal-regulated kinase; GSB-106, bis-(N-monosuccinyl-L-seryl-L-lysine) hexamethylenediamide; GSB-2|4, bis-(N-monosuccinyl-L-methionyl-L-serine) heptamethylenediamide; SD, standard deviation.

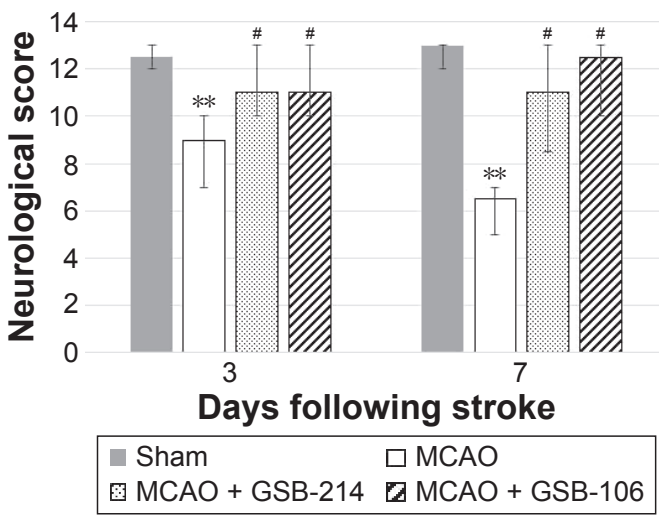

Figure 4 Dimeric dipeptides GSB-106 and GSB-2I4 improved the performance of rats on the limb-placing test after MCAO.

Notes: The data are presented as medians with interquartile ranges. $* * P<0.0$ I vs the sham-operated group. ${ }^{\# P}<0.05$ vs the MCAO group (Mann-Whitney U-test with Bonferroni's correction).

Abbreviations: GSB-106, bis-(N-monosuccinyl-L-seryl-L-lysine) hexamethylenediamide; GSB-2I4, bis-( $\mathrm{N}$-monosuccinyl-L-methionyl-L-serine) heptamethylenediamide; MCAO, middle cerebral artery occlusion.

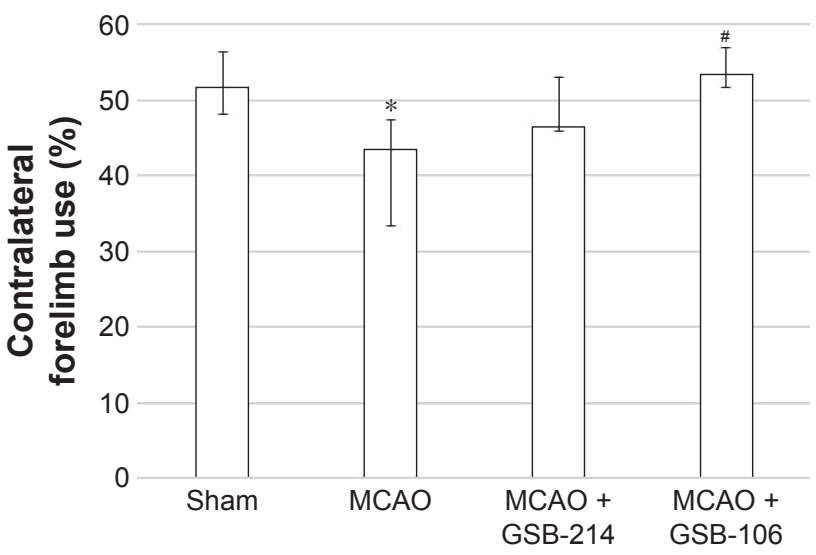

Figure 5 GSB-I06 treatment completely abolished forelimb asymmetry on the cylinder test after MCAO. Data are presented as medians with interquartile ranges. Notes: $* P<0.05$ vs the sham-operated group. ${ }^{* P}<0.05$ vs the MCAO group (MannWhitney U-test with Bonferroni's correction).

Abbreviations: GSB-106, bis-(N-monosuccinyl-L-seryl-L-lysine) hexamethylenediamide; GSB-2|4, bis-( $\mathrm{N}$-monosuccinyl-L-methionyl-L-serine) heptamethylenediamide; MCAO, middle cerebral artery occlusion. 


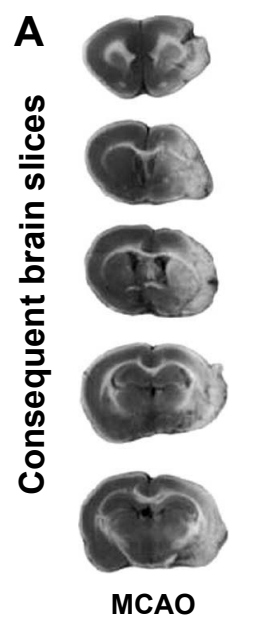

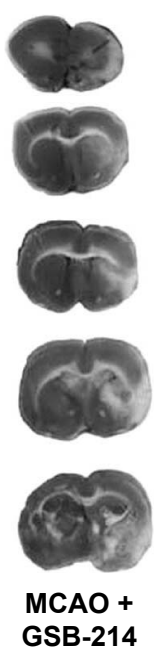

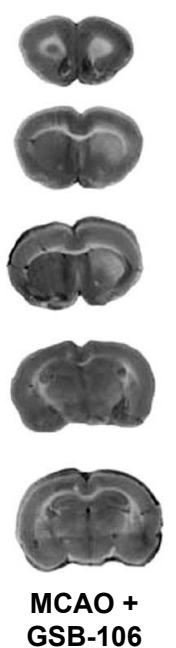

B

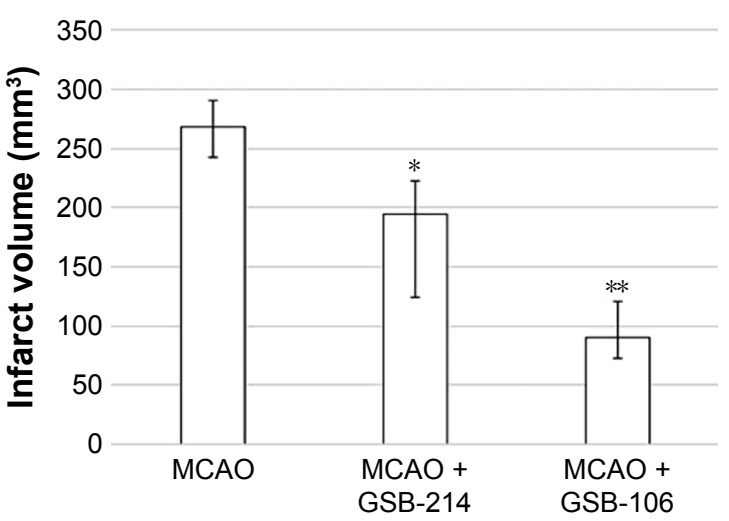

Figure 6 Dimeric dipeptides GSB-106 and GSB-2I 4 decrease infarct volume after MCAO.

Notes: (A) Representative TTC-stained brain slices. (B) The dimeric dipeptides GSB-I06 and GSB-2I 4 decreased cerebral infarct volume 7 days after stroke. Morphometric measurements of infarct volume were performed using TTC staining. The data are presented as medians with interquartile ranges. $* P<0.05, * * P<0.0 \mathrm{I}$ vs the $M C A O$ group (Mann-Whitney U-test with Bonferroni's correction).

Abbreviations: GSB-106, bis-(N-monosuccinyl-L-seryl-L-lysine) hexamethylenediamide; GSB-2I4, bis-(N-monosuccinyl-L-methionyl-L-serine) heptamethylenediamide; MCAO, middle cerebral artery occlusion; TTC, 2,3,5-triphenyltetrazolium chloride.

As we reported earlier, GSB-106 exhibited antidepressant activity on the forced swimming test in mice, whereas GSB-214 did not. ${ }^{13}$ These results indicated that the antidepressant activity of BDNF mimetics was associated with activation of both the PI3K/AKT and MAPK/ERK signaling cascades, whereas selective PI3K/AKT pathway activation did not produce antidepressant-like effects. The association of the MAP-kinase pathway with antidepressant activity could also be explained by the central role of this signaling pathway in the regulation of mechanisms of neurogenesis and neuroplasticity, impairments of which are considered to be among the main etiopathological factor of depression. ${ }^{23}$ Thus, our results demonstrated the importance of MAP kinase pathway activation to the therapeutic efficacy of BDNF dimeric dipeptide mimetics.

\section{Conclusion}

The results presented in this paper supported the beneficial effects of the BDNF loop 4 dimeric dipeptide mimetic GSB106 in reducing cerebral infarct volume and improving neurological function after stroke, thereby suggesting a potential role for the dipeptide as a therapeutic agent.

\section{Acknowledgment}

This work was supported by the Russian Science Foundation (project 14-15-00596).

\section{Disclosure}

The authors report no conflicts of interest in this work.

\section{References}

1. Wu D. Neuroprotection in experimental stroke with targeted neurotrophins. NeuroRx. 2005;2(1):120-128.

2. Yu SJ, Tseng KY, Shen H, Harvey BK, Airavaara M, Wang Y. Local administration of AAV-BDNF to subventricular zone induces functional recovery in stroke rats. PLoS One. 2013;8(12):e81750.

3. Ploughman M, Windle V, MacLellan CL, White N, Doré JJ, Corbett D. Brain-derived neurotrophic factor contributes to recovery of skilled reaching after focal ischemia in rats. Stroke. 2009;40(4): 1490-1495.

4. Berretta A, Tzeng YC, Clarkson AN. Post-stroke recovery: the role of activity-dependent release of brain-derived neurotrophic factor. Expert Rev Neurother. 2014;14(11):1335-1344.

5. Chen A, Xiong LJ, Tong Y, Mao M. The neuroprotective roles of BDNF in hypoxic ischemic brain injury. Biomed Rep. 2013;1(2):167-176.

6. Jiang Y, Wei N, Zhu J, et al. Effects of brain-derived neurotrophic factor on local inflammation in experimental stroke of rat. Mediators Inflamm. 2010;2010:372423.

7. Schäbitz WR, Steigleder T, Cooper-Kuhn CM, et al. Intravenous brainderived neurotrophic factor enhances poststroke sensorimotor recovery and stimulates neurogenesis. Stroke. 2007;38(7):2165-2172.

8. Guan J, Tong W, Ding W, et al. Neuronal regeneration and protection by collagen-binding BDNF in the rat middle cerebral artery occlusion model. Biomaterials. 2012;33(5):1386-1395.

9. Yoshii A, Constantine-Paton M. Post-synaptic BDNF-TrkB signaling in synapse maturation, plasticity and disease. Dev Neurobiol. 2010;70(5): 304-322.

10. Kaplan DR, Miller FD. Neurotrophin signal transduction in the nervous system. Curr Opin Neurobiol. 2000;70(5):381-391.

11. Jiang P, Zhu T, Xia Z, et al. Inhibition of MAPK/ERK signaling blocks hippocampal neurogenesis and impairs cognitive performance in prenatally infected neonatal rats. Eur Arch Psychiatry Clin Neurosci. 2015; 265(6):497-509.

12. Tyler WJ, Alonso M, Bramham CR, Pozzo-Miller LD. From acquisition to consolidation: on the role of brain-derived neurotrophic factor signaling in hippocampal-dependent learning. Learn Mem. 2002;9(5): 224-237.

13. Gudasheva TA, Tarasiuk AV, Pomogaibo SV, et al. Design and synthesis of dipeptide mimetics of brain-derived neurotrophic factor. Bioorg Khim. 2012;38(3):280-290. 
14. Senger DL, Campenot RB. Rapid retrograde tyrosine phosphorylation of trkA and other proteins in rat sympathetic neurons in compartmented cultures. J Cell Biol. 1997;138(2):411-421.

15. Longa EZ, Weinstein PR, Carlson S, Cummins R. Reversible middle cerebral artery occlusion without craniectomy in rats. Stroke. 1989; 20(1):84-91.

16. Jolkkonen J, Puurunen K, Rantakömi S, Harkonen A, Haapalinna A, Sivenius J. Behavioral effects of the alpha(2)-adrenoreceptor antagonist, atipamezole, after focal cerebral ischemia in rats. Eur J Pharmacol. 2000;400(2-3):211-219.

17. De Ryck M, Van Reempts J, Borgers M, Wauquier A, Janssen PA. Photochemical stroke model: flunarizine prevents sensoriomotor deficits after neocortical infarcts in rats. Stroke. 1989;20(10):1383-1390.

18. Schallert T, Woodlee MT, Fleming SM. Disentangling multiple types of recovery from brain injury. In: Krieglstein J, editor. Pharmacology of Cerebral Ischemia. Medpharm Scientific Publishers Stuttgart; 2002: 201-216.

19. Gudasheva TA, Povarnina PY, Antipova TA, Firsova YN, Konstantinopolsky MA, Seredenin SB. Dimeric dipeptide mimetics of the nerve growth factor Loop 4 and Loop 1 activate TRKA with different patterns of intracellular signal transduction. J Biomed Sci. 2015; $22: 106$.
20. Zhang Y, Pardridge WM. Blood-brain barrier targeting of BDNF improves motor function in rats with middle cerebral artery occlusion. Brain Res. 2006;1111(1):227-229.

21. Seredenin SB, Silachev DN, Gudasheva TA, Pirogov YA, Isaev NK. Neuroprotective effect of GK-2, a dipeptide mimetic of nerve growth factor, during experimental focal ischemia in middle cerebral artery basin. Bull Exp Biol Med. 2011;151(5):584-587.

22. Gudasheva TA, Povarnina PLU, Seredenin SB. Dipeptide mimetic of the brain derived neurotrophic factor prevents the stress-induced impairments in hippocampus-dependent neurogenesis in mice. Bull Exp Biol Med. In press 2016.

23. Wainwright SR, Galea LA. The neural plasticity theory of depression: assessing the roles of adult neurogenesis and PSA-NCAM within the hippocampus. Neural Plast. 2013;2013:805497.

\section{Publish your work in this journal}

Drug Design, Development and Therapy is an international, peerreviewed open-access journal that spans the spectrum of drug design and development through to clinical applications. Clinical outcomes, patient safety, and programs for the development and effective, safe, and sustained use of medicines are the features of the journal, which has also been accepted for indexing on PubMed Central. The manuscript management system is completely online and includes a very quick and fair peer-review system, which is all easy to use. Visit http://www.dovepress.com/testimonials.php to read real quotes from published authors.

Submit your manuscript here: http://www.dovepress.com/drug-design-development-and-therapy-journal 